\title{
DEFORMACIÓN SISMOTECTÓNICA A PARTIR DE MECANISMOS FOCALES DE TERREMOTOS EN EL VALLE DEL CAUCA, SUROCCIDENTE DE COLOMBIA
}

\author{
SEISMOTECTONIC DEFORMATION FROM FOCAL MECHANISMS OF \\ EARTHQUAKES IN THE VALLE DEL CAUCA, SOUTHWESTERN COLOMBIA
}

\author{
Elkin de J. Salcedo ${ }^{1,2 *}$ y Jhon L. Pérez ${ }^{2,3}$ \\ ${ }^{1}$ Departamento de Geografía, Universidad del Valle, Cali, Colombia \\ ${ }^{2}$ Grupo GEORIESGOS - Observatorio Sismológico y Geofísico del \\ Suroccidente Colombiano, Universidad del Valle, Cali. \\ ${ }^{3}$ Posgrado en Ciencias de la Tierra, Departamento de Sismología, CICESE \\ *Autor para contacto: elkin.salcedo@correounivalle.edu.co
}

(Recibido: 24/12/2016; aceptado: 10/03/2017)

\begin{abstract}
Using data of focal mechanisms, we analyzed the seismotectonic strain regime in the Valle del Cauca, of southwestern Colombia, 49 earthquakes were selected with magnitude $\mathrm{Mw} \geq 4.8$ within the period of 1976 to 2010. This data was taken from ISC (International Seismological Center) and GCMT (Global Centroid Moment Tensor) database. According with the spatial distribution of different types of focal mechanisms, we recognize four main seismotectonic deformation zones: the seaward side of the trench (Environment-I), the bending and interplate interaction zone (Environment-II), the shallow continental zone (Environment-III), and the intermediate Benioff zone (Environment-IV). Overall, the geodynamic features of these environments are controlled by the subduction zone. We obtained that the bending zone has an angle of inclination of $30^{\circ}$, which generates a tangential deformation stress $\left(\tau_{s}\right)$ with a magnitude of $7.67 * 10^{22} \mathrm{~Pa}$; a tangential force (Fs) of $1.06 * 10^{27} \mathrm{~N} / \mathrm{m}$, and a normal force (Fn) equivalent to $1.47 * 10^{28} \mathrm{~N} / \mathrm{m}$ with a slab pull force of $1.57 * 10^{28} \mathrm{~N} / \mathrm{m}$. Moreover, the interplate seismic coupling coefficient is 0.21 , suggesting a subduction zone of intermediate activity. On the other hand, the dislocation velocity (vs) and subsidence velocity (vd) of the oceanic plate are $3.68 \mathrm{~cm} /$ year and $2.76 \mathrm{~cm} /$ year, respectively. These geodynamic and kinematic characteristics explain the frequent seismic events of small to moderate intensity $(2 \leq \mathrm{M} \leq 5)$, and the occurrence of a possible earthquake with $\mathrm{Mw} \geq 7$ magnitude in the region.
\end{abstract}

Keywords: Earthquakes, focal mechanisms, seismotectonic deformation, Valle del Cauca, southwestern Colombia.

RESUMEN: Se analiza el régimen de deformación sismotectónica en el Valle del Cauca, suroccidente de Colombia, usando datos de mecanismos focales de 49 terremotos con magnitud $\mathrm{Mw} \geq 4,8$ en el periodo 1976-2010, tomados de la base de datos del ISC (International Seismological Center) y del GCMT (Global Centroid Moment Tensor). Se considera la distribución de los tipos de mecanismos focales, reconociendo cuatro ambientes de deformación sismotectónica: Zona 
antes de la fosa (Ambiente-I), Zona de flexión e interacción interplacas (Ambiente-II), Zona continental superficial (Ambiente-III) y Zona de Benioff intermedia (Ambiente-IV). En términos generales, las características geodinámicas de estos ambientes están gobernadas por la subducción de la placa oceánica bajo la continental. Se estimó un ángulo de inclinación de $30^{\circ}$ para la zona de flexión, generando un esfuerzo tangencial deformante $\left(\tau_{s}\right)$ de $7,67 * 10^{22} \mathrm{~Pa}$, una fuerza tangencial (Fs) de $1,06 * 10^{27} \mathrm{~N} / \mathrm{m}$, una fuerza normal (Fn) equivale a $1,47 * 10^{28} \mathrm{~N} / \mathrm{m}$ y una fuerza de arrastre de la placa subducida de $1,57^{*} 10^{28} \mathrm{~N} / \mathrm{m}$. El coeficiente de acoplamiento sísmico interplaca es de 0,21 , lo que sugiere una zona de subducción de actividad intermedia. Por otro lado, la velocidad de dislocación y de hundimiento de la placa oceánica, respectivamente, tienen valores de vs $=3,68 \mathrm{~cm} /$ año y vd $=2,76 \mathrm{~cm} /$ año. Estas características geodinámicas y cinemáticas explican la frecuente ocurrencia de eventos sísmicos de pequeños a moderados $(2 \leq \mathrm{M} \leq 5)$, así como la ocurrencia de un posible sismo de magnitud $\mathrm{Mw} \geq 7$ en la región.

Palabras clave: Terremotos, mecanismos focales, deformación sismotectónica, Valle del Cauca, suroccidente colombiano.

\section{INTRODUCCIÓN}

Para avanzar en la solución del problema de la amenaza y el riesgo sísmico en la región del Valle del Cauca es fundamental tener un amplio conocimiento de las particularidades de las fuentes sismogénicas que existen en la zona y su grado de actividad. De la misma manera, es necesario conocer las características del régimen sismotectónico, entendiéndose éste como la distribución espacio-temporal de la sismicidad, por un lado, y el análisis de los mecanismos focales que gobiernan la región, la caracterización del campo de esfuerzos y el régimen de deformación actual asociado, por otro lado.

De acuerdo con Nivia et al. (1997), los esfuerzos dominantes en el Valle del Cauca han producido un sistema complejo de fallas regionales en el que se identifican tres direcciones dominantes de fallamiento: uno en dirección $\mathrm{N} 20^{\circ}-30^{\circ} \mathrm{E}$, otro en dirección $\mathrm{N} 60^{\circ}-70^{\circ} \mathrm{E}$, y el último en dirección $\mathrm{N} 40^{\circ}-50^{\circ} \mathrm{W}$, determinando que los movimientos que se generan a lo largo de este conjunto de fallas reacomodan regionalmente los esfuerzos que se producen por la interacción de las placas tectónicas Nazca, Sudamericana y Caribe, dando lugar a traslación y rotación de bloques corticales. De esta manera, los esfuerzos tectónicos dominantes en la región han sufrido diversas etapas de inversión, generando complejos procesos de deformación en la zona (López, 2006; París et al., 1992).
El complejo sistema tectónico regional genera fallas geológicas de diversos tipos. Sin embargo, predominan fallas similares a las encontradas en sistemas de colisión que se caracterizan por tener componentes de desplazamiento lateral, resultante de una tectónica relacionada con la subducción oblicua (Asped, 1984; Navia, 2001). Además, en la región se logra evidenciar diversos sitios donde la cinemática refleja la incidencia de esfuerzos tectónicos en dirección E-W, lo cual explica la existencia de fallamiento en dirección NW (López, 2006).

Este sistema complejo de fallamiento junto con la zona de subducción que resulta de la interacción de las placas Nazca y Sudamérica constituyen las principales fuentes sismogénicas de la región, siendo responsables de la ocurrencia de sismos históricos y recientes que han afectado diversas poblaciones del Valle del Cauca (Martínez, 2015).

Así, considerando la complejidad tectónica y la manifestación de la actividad sísmica que se presenta en la región, se plantea la necesidad de analizar con mayor rigor las particularidades de la sismicidad, el régimen de esfuerzos dominantes y las actuales condiciones de deformación sismotectónica.

En el presente trabajo se realiza el análisis de la deformación sismotectónica que se produce en la región del Valle del Cauca con la ocurrencia de los terremotos. Para ello, son usados 
datos de mecanismos focales de terremotos con magnitud $\mathrm{Mw} \geq 4,8$ ocurridos en el periodo entre 1976-2010, tomados de la base de datos del ISC (International Seismological Center) y del Global Centroid Moment Tensor (GCMT). Con base en el análisis de la información anterior, la zona de estudio fue subdividida en cuatro ambientes tectónicos, a los que se les calculó el tensor de deformación, la tasa de velocidad de deformación sísmica y algunos parámetros dinámicos importantes de la zona de subducción.

\section{MARCO TECTÓNICO}

La región del Valle del Cauca (suroccidente de Colombia), se ubica donde la placa Nazca (corteza oceánica) subduce debajo de la placa de Sudamérica (Fig. 1). La placa Nazca se desplaza en dirección ENE con movimiento relativo entre $50 \mathrm{~mm} /$ año y $78 \mathrm{~mm} /$ año (Freymueller et al., 1993; Kellogg et al., 1983; Pennington, 1981; Trenkamp et al., 2002, Trenkamp et al., 2004), conservando sus propiedades mecánicas hasta ser consumida en el manto debajo del continente suramericano. Las placas Nazca y Suramérica interactúan en la zona de la Fosa Colombo-Ecuatoriana que recorre el fondo marino más o menos paralelo a la costa, definiendo el límite superficial de la zona de subducción.

Las estructuras tectónicas presentes en la zona occidental de Colombia son el producto de la interacción y subducción de las placas Nazca y Sudamericana. Para esta zona se presume la existencia de al menos tres segmentos tectónicos los cuales se caracterizan por su propio régimen sísmico. Estos segmentos son (Arcila y Dimaté, 2005; Collot et al., 2004; Corredor, 2003; Monsalve, 1998; Pennington, 1981; Pedraza, 2006; Fig. 1): "Segmento norte", que representa la subducción del bloque Panamá-Chocó bajo el extremo noroeste de Colombia. Está orientado a $310^{\circ}$ de azimut y su plano de Benioff buza de $25^{\circ}$ hacia los $40^{\circ}$, la fosa o trinchera en este segmento tiene una longitud de $170 \mathrm{~km}$ y la magnitud máxima estimada para un sismo aquí es de $7,8 \mathrm{Mw} ; 2$ ) el "Segmento Centro", está orientado a $130^{\circ}$ de azimut y su plano de Benioff buza de $40^{\circ}$ hacia los $110^{\circ}$, la fosa tiene una longitud de $160 \mathrm{~km} \mathrm{y}$ la magnitud máxima estimada para un sismo también es de 7,8 Mw; 3) el "Segmento sur", ubicado frente a la costa sur de la región del Valle del Cauca y norte de Ecuador, está orientado a $40^{\circ}$ de azimut y su plano de Benioff buza de $30^{\circ}$ hacia $\operatorname{los} 130^{\circ}$, la fosa en este segmento tiene una longitud de $550 \mathrm{~km}$. La magnitud máxima calculada para un sismo aquí es de $8,8 \mathrm{Mw}$.

Respecto al fallamiento cortical, la interacción de las placas Nazca y Sudamericana producen un régimen de esfuerzos tectónicos regional que en el Valle del Cauca genera un sistema complejo de fallas (Nivia, 2001; Nivia et al., 1997) como se puede ver en la figura 2 .

\section{DEFORMACIÓN SISMOTECTÓNICA}

La ocurrencia de terremotos resulta de la dislocación repentina (ruptura) de una falla, que produce a su vez, un patrón de radiación energético o momento sísmico. La información de la solución del plano de falla y la orientación geométrica del tensor de momento sísmico, pueden ser proporcionadas a partir del mecanismo focal de un terremoto. Cuantitativamente se puede estimar la contribución de la magnitud de los momentos sísmicos a la deformación que se acumula en una zona tectónica determinada, considerando para ello el área de la fuente sismogénica, el momento sísmico conocido de un conjunto de terremotos y el rango de tiempo en el cual estos ocurren.

La deformación sísmica en un volumen se puede relacionar con el tensor de deformación en la región y la suma del tensor momento de los terremotos que ocurren dentro de ese volumen de la siguiente manera (Kostrov, 1974; Risnichenko, 1976; Risnichenko, 1985):

$$
\dot{\varepsilon}_{i j}=\frac{1}{2 \mu \Omega} \frac{\sum_{n=1}^{N} M_{i j}}{T}
$$




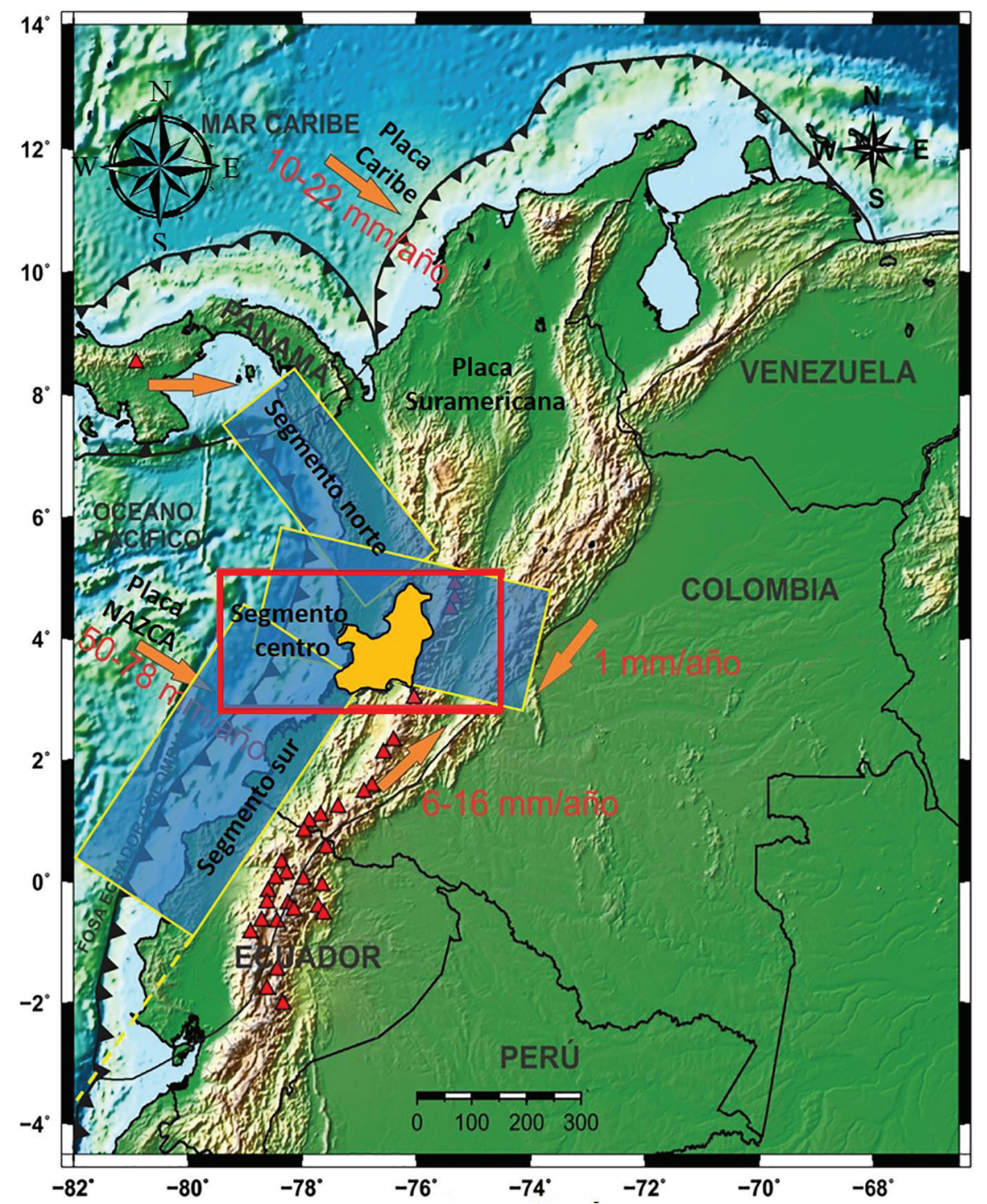

Fig. 1: Características tectónicas de la zona de convergencia entre la placa Nazca y Sudamericana en Colombia. Los valores de movimiento relativo de las placas son de acuerdo a los datos GPS. Los rectángulos en azul corresponden a la segmentación de la zona de subducción; el rectángulo en rojo indica la zona de estudio; el polígono naranja corresponde a la región del Valle del Cauca y los triángulos en rojo a los volcanes activos.

Donde es el tensor de deformación, $\Omega$ es el volumen deformado debido a la actividad sísmica, $M_{i j}$ son las componentes del tensor momento sísmico para todos los terremotos que ocurren dentro de este volumen en el tiempo $T$ en años, $\mu$ es el módulo de rigidez o la viscosidad del medio dependiendo del ambiente sismotectónico y, $N$ es el número de terremotos. 


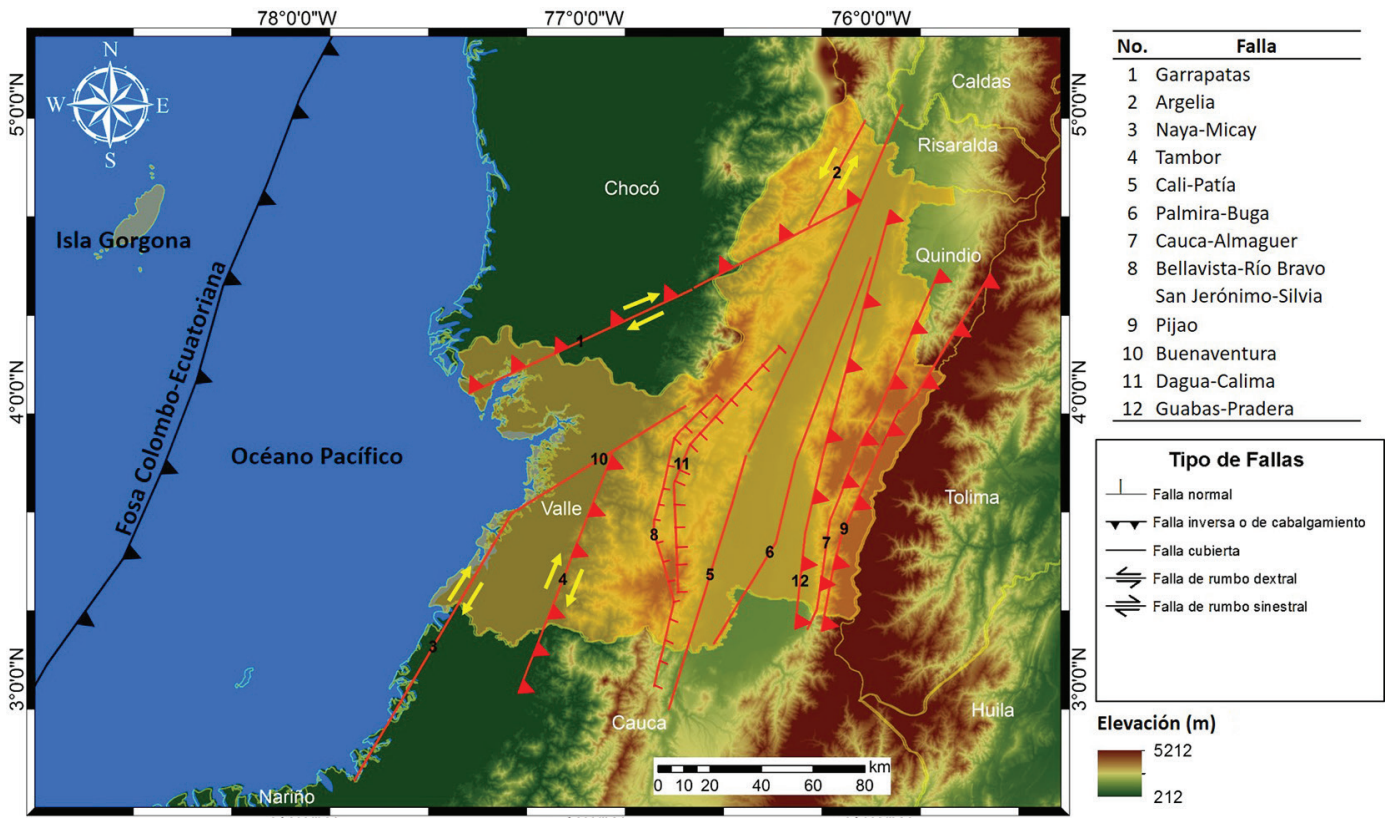

Fig. 2: El polígono en color naranja corresponde al departamento del Valle del Cauca y las líneas rojas a las principales fallas de esta región (Modificado de USGS, 2000; Nivia, 2001).

\section{METODOLOGÍA}

En este trabajo se analiza la deformación sismotectónica en la región del Valle del Cauca, producida por la interacción y subducción de las placas Nazca y Sudamericana. Como lo proponen Salcedo y Pérez (2016), la zona de estudio es subdividida en cuatro ambientes tectónicos: Ambiente I - Lado marino de fosa, Ambiente II - Zona de flexión e interacción interplacas, Ambiente III - Zona continental superficial y Ambiente IV - Zona de Benioff intermedia. En cada una de estos ambientes se calcula el tensor de deformación y la tasa de velocidad de la deformación sísmica. Además, se calculan los parámetros dinámicos en la zona de subducción.

\section{Deformación Sismotéctonica}

Para el análisis se usa el concepto de flujo sísmico de la masa rocosa propuesto inicialmente por Risnichenko (1965a) cuyo significado físico, en términos de la mecánica del medio continuo, fue establecido por Kostrov (1974). El método establece que los parámetros dinámicos del foco sísmico generalmente están correlacionados con la dirección de los ejes de deformación determinados por el mecanismo focal (Brune, 1968; Aki y Richards, 1980) dentro de un volumen deformado (Risnichenko, 1976; Risnichenko y Jibladze, 1976).

La deformación sísmica es calculada a partir de la ecuación (1). Para un ambiente visco-plástico, como la zona sismoactiva en una placa subducente, la ecuación (1) puede reescribirse como (Heuret et al., 2011; Risnichenko, 1985):

$$
\dot{\varepsilon}_{i j}=\frac{1}{2 \mu T W L} \sum_{n=1}^{N} M_{i j}
$$

Donde $\mu$ corresponde al módulo de rigidez o de viscosidad del medio (Kanamori y Astiz, 1985; Heuret et al., 2011), T es el tiempo de observación 
de los terremotos considerados, $W$ es la extensión en profundidad de la zona inclinada y L es la longitud del segmento de la fosa a estudiar. Estos parámetros, caracterizados por Heuret et al. (2011), describen la fuente sismogénica en la región de interacción de las dos placas.

De acuerdo con Brune (1968) y Scholz (1994), se considera que la tasa de dislocación puede determinarse mediante la sumatoria de los momentos sísmicos, calculando el aporte individual de cada uno de los sismos en el movimiento general de la falla o del bloque deformado. Así, según Risnichenko (1965b), si se quiere establecer la tendencia de la deformación tectónica predominante en un elemento de volumen de la masa rocosa, es necesario el análisis de los mecanismos focales; por lo tanto, cada sismo que ocurra dentro de un bloque actúa como un "contador" de la dislocación acumulada en el tiempo considerado, por lo cual puede usarse desde un solo evento hasta un número $\mathrm{N}$ de sismos (Kuchay, 1990).

La velocidad media de deformación del volumen se determina a partir de la orientación del campo de esfuerzos (Guzmán-Speziale, 2001), usando la siguiente ecuación:

$$
V_{i j}=l_{i j} \dot{\varepsilon}_{i j}
$$

Donde $l_{1}, l_{2}$ y $l_{3}$ son las dimensiones del volumen a lo largo de las direcciones $x, y$ y $z$, que en el sistema de coordenadas cartesianas se orientan de tal manera que $x$ está en la dirección norte, y hacia el este y $z$ hacia el centro de la Tierra.

La magnitud promedio $M w$ en cada ambiente tectónico puede ser obtenida usando la relación de Kanamori (1977), reescrita de la siguiente forma:

$$
M w=2 / 3 \log (\mu \Omega T \varepsilon)-10,7
$$

Donde $\Omega$ es el volumen deformado considerado y $\varepsilon$ es el máximo valor propio (eigenvalue) del tensor de deformación. También se puede calcular el promedio del deslizamiento de la ruptura (slip) del sismo de magnitud $M w$ para cada volumen sismogénico aplicando la relación de Kanamori y Astiz (1985), expresada como:

$$
\bar{D}=\xi 10^{0.5 M w+5,37}
$$

Donde la constante $\xi=1,1 * 10^{-7} \operatorname{dinas}^{-1 / 3} \mathrm{~cm}^{2 / 3}$.

\section{Dinámica de la zona de subducción}

Las velocidades relacionadas con el movimiento del conjunto placas se derivan de la regla del paralelogramo (Lobkovsky, 1988). A la velocidad de dislocación $\left(v_{s}\right)$, con tendencia paralela hacia la zona plástica, se sobrepone la velocidad horizontal de la placa $\left(v_{l}\right)$. Como resultado de la suma de estas velocidades, en el límite de la región plástica aparece la velocidad $\left(v_{d}\right)$ que representa el movimiento descendente de la placa subducente (Figura 3).

La velocidad $\left(v_{d}\right)$ se expresa mediante la siguiente relación.

$$
v_{d}=v_{l}+v_{s}
$$

Las magnitudes absolutas de la velocidad de dislocación $\left(v_{s}\right)$ y de hundimiento de la placa oceánica $\left(v_{d}\right)$ con respecto a la velocidad horizontal de avance $\left(v_{l}\right)$, se expresan por las ecuaciones (7) y 8) (Lobkovsky, 1988; Capitanio et al., 2007):

$$
\begin{aligned}
& v_{s}=v_{t} \operatorname{sen} \beta / \operatorname{sen}(\alpha+\beta) \\
& v_{d}=v_{t} \operatorname{sen} \alpha / \operatorname{sen}(\alpha+\beta)
\end{aligned}
$$

Donde $\alpha$ y $\beta$ corresponden a los ángulos de inmersión y de doblez de la placa subducente, respectivamente. La velocidad del movimiento horizontal de la placa $\left(v_{l}\right)$, se puede determinar a partir de datos sismológicos o geodésicos.

Las fuerzas que actúan en la zona plástica, corresponden a la tangencial $\left(F_{s}\right)$ y normal $\left(F_{n}\right)$ (Figura 4). Estas pueden ser expresadas en función del espesor $\left(H_{l}\right)$ y el ángulo de inmersión de la placa $(\alpha)$ considerando a su vez los correspondientes esfuerzos tangencial $\left(\tau_{s}\right)$ y normal $\left(\sigma_{n}\right)$, respectivamente (Lobskovky, 1988).

$$
F_{s}=\tau_{s} H_{l} / \operatorname{sen} \alpha
$$




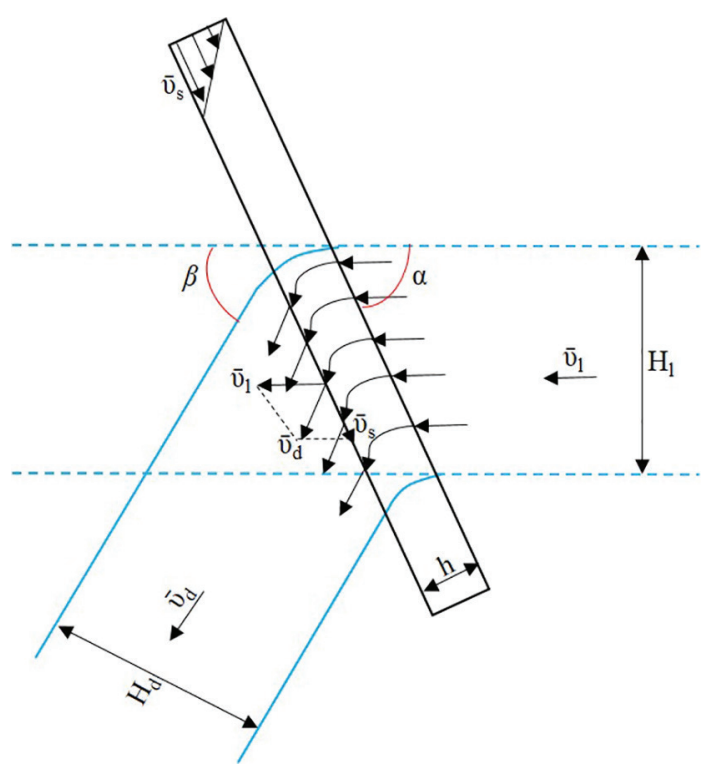

Fig. 3: Esquema de la relación de velocidad y deformación en la zona de doblez de una placa tectónica en subducción (Modificado de Lobskovky, 1988).

$$
F_{n}=\sigma_{n} H_{l} / \operatorname{sen} \alpha
$$

En este caso $\tau_{s}$ está dado por la relación:

$$
\tau_{i j}=2 \mu \dot{\varepsilon} \quad i j
$$

Donde $\dot{\varepsilon}_{i j}$ es la tasa de deformación resultante entre las componentes horizontal $\left(\dot{\varepsilon}_{y y}\right)$ y vertical ( $\dot{\varepsilon}_{z z}$ ), y $\mu$ es el coeficiente de viscosidad equivalente a $6,5^{*} 10^{23} \mathrm{~Pa} / \mathrm{seg}$. Y $\sigma_{n}$ se expresa por la ley de Amonton (Turcotte y Schubert, 1982):

$$
\sigma_{n}=\tau_{s}(1+\cos 2 \alpha) / \operatorname{sen} 2 \alpha
$$

La fuerza de arrastre ejercida por la placa oceánica fría y densa al sumergirse en el manto debido a su propio peso (Conrad et al., 2004) es la misma fuerza resultante $R$ que determina la condición en que se subduce la placa (Figura 3), la cual se expresa de la siguiente manera (Lobskovky, 1988):

$$
R=F_{s}+F_{n}
$$

La correlación de los parámetros geométricos y dinámicos entre diversas zonas de subducción han sido estudiadas por varios autores, prestando especial interés en el balance de fuerzas e incluyendo la fuerza de fricción en la zona de interacción de las placas (Kanamori, 1986; Jarrard, 1986; Pacheco et al., 1993; Kawasaki et al., 2001; Conrad et al., 2004; Heuret et al., 2011).

\section{DATOS}

En el presente estudio se usaron datos de mecanismos focales de terremotos con magnitud $\mathrm{Mw}$ $\geq 4.8$, ocurridos en el periodo entre 1976-2012 en la región del Valle del Cauca comprendida entre las coordenadas $2,5^{\circ}$ y $5,3^{\circ}$ de latitud Norte y $75,6^{\circ}$ y $79,5^{\circ}$ de longitud Oeste. Los datos se tomaron de los catálogos del ISC (International Seismological Center) y del GCMT (Global Centroid Moment Tensor) (Dziewonski, 1982, Dziewon ski et al., 1981, Dziewonski y Woodhouse, 1983, Dziewonski et al., 1983).

La información extraída corresponde a la localización de los eventos, magnitud $M w$, planos nodales, ejes de deformación P y T (Cuadro 1). Además, se obtuvieron las componentes del tensor momento sísmico y el momento sísmico escalar Mo. En total se reunieron 49 eventos sísmicos, que se distribuyen en los ambientes tectónicos señalados (Figura 5): Ambiente I - Lado marino de la fosa, Ambiente II - Zona de flexión e interacción interplacas, Ambiente III - Zona continental superficial y Ambiente IV - Zona de Benioff intermedia.

Los ambientes tectónicos indicados se caracterizan de la siguiente manera:

\section{Ambiente Tectónico I: zona del lado marino de la Fosa Colombo-Ecuatoriana}

Este ambiente se definió en la zona donde se presentan los eventos localizados antes la fosa (Figura 5), limitada entre las coordenadas $4,99^{\circ}$ y $2,51^{\circ}$ de latitud Norte y $79,82^{\circ}$ y $78.08^{\circ}$ de longitud Oeste. Cubre un área equivalente a 19552 $\mathrm{km}^{2}$ y la profundidad de los sismos está entre 12 y $28 \mathrm{~km}$. El volumen de este ambiente equivale a 


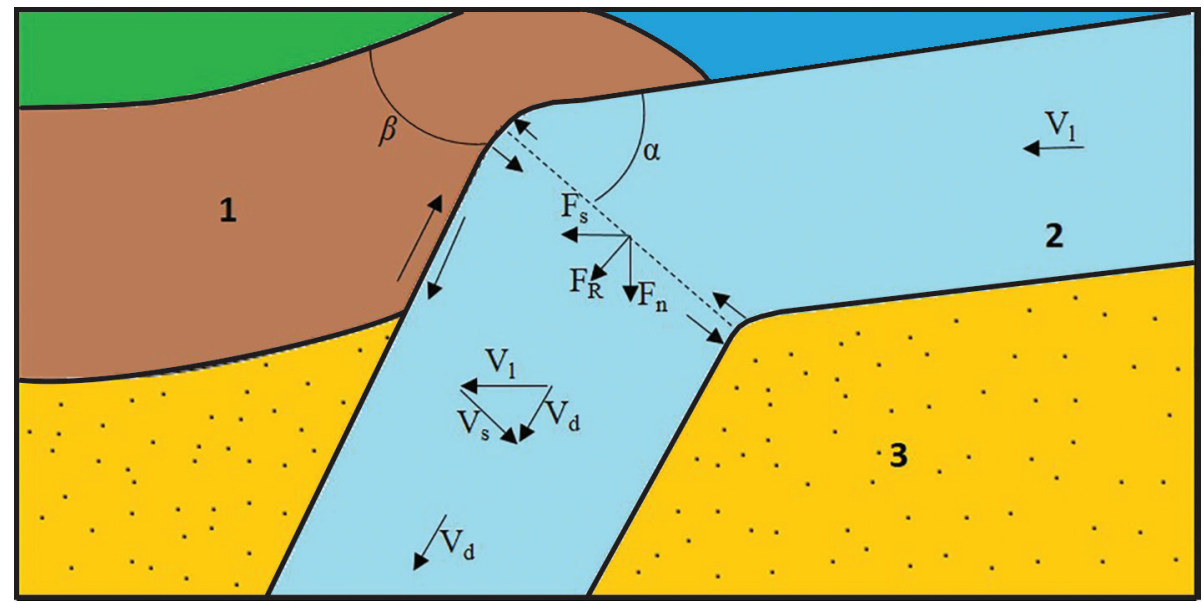

Fig. 4: Modelo mecánico de la deformación de la placa en la zona de subducción. 1- Litosfera oceánica subducente, 2- placa continental, 3- astenosfera (Modificado de Lobskovky, 1988).

$332387 \mathrm{~km}^{3}$. Los datos de la solución del mecanismo focal de los terremotos de la zona se presentan en el cuadro 1. La numeración en la tabla corresponde al número de cada uno de los eventos presentados en la Figura 5.

\section{Ambiente Tectónico II: Zonas de Flexión y de interacción interplacas}

\section{Zona de flexión de la placa subducente}

La geometría de la placa subducente es bien definida por la zona de Benioff, misma que representa el ángulo con el cual la placa quiebra o flexiona para introducirse en el manto. El ángulo de inmersión de la placa incrementa gradualmente desde la fosa hasta cerca de $80-150 \mathrm{~km}$ de profundidad y se mantiene casi constante por debajo de esta profundidad. El mayor cambio en la inmersión, caracteriza el ángulo de la placa hasta una profundidad de $60 \mathrm{~km}$ (ángulo de inmersión superficial o punto de quiebre), luego este ángulo cambia hasta una profundidad de $100 \mathrm{~km}$ (ángulo de inmersión intermedio) y, finalmente, el ángulo de inmersión profundo en el intervalo entre 100 y 400 km (Jarrard, 1986).
El ángulo de inmersión superficial o ángulo de quiebre de la placa Nazca en la zona de subducción en el territorio colombiano es $\alpha=22^{\circ}$ Jarrard (1986). Mientras que el ángulo de subducción de la placa $(\beta)$ calculado en el presente trabajo es aproximadamente $30^{\circ}$.

\section{Zona de interacción interplacas}

La zona de interacción o de acople de las placas, producida por la fuerte convergencia entre las placas oceánica y continental, corresponde a una amplia zona de deformación donde se producen los más grandes terremotos, que liberan la mayor parte de la energía allí acumulada (Figura 6). En esta zona la placa subducente se mueve con una velocidad que supera la fuerza de fricción entre las dos placas (Conrad et al., 2004).

En la esquematización geométrica mostrada en la Figura 6 , se asume que $\left(U_{x} ; U_{z}\right)$ y $\left(D_{x} ; D_{z}\right)$ corresponden a las coordenadas de los límites superior e inferior, respectivamente, de la inclinación de la zona sismogénica. Así, la extensión en profundidad de la zona inclinada $\mathrm{W}$ está dada por la distancia entre $U$ y $D$, y el ángulo de inclinación 
Cuadro 1

Parámetros de los mecanismos focales de los sismos localizados en los cuatro ambientes tectónicos estudiados, periodo 1976 - 2012.

\begin{tabular}{|c|c|c|c|c|c|c|c|c|c|c|c|c|}
\hline \multirow{3}{*}{ No. } & \multirow{3}{*}{$\begin{array}{c}\text { Fecha } \\
\text { dd:mm:aa }\end{array}$} & \multirow{2}{*}{\multicolumn{3}{|c|}{ Localización }} & \multirow{3}{*}{$\begin{array}{c}\text { Mag. } \\
\mathrm{Mw}\end{array}$} & \multirow{2}{*}{\multicolumn{3}{|c|}{ Plano de falla }} & \multicolumn{4}{|c|}{ Ejes de deformación* } \\
\hline & & & & & & & & & \multicolumn{2}{|c|}{$\mathrm{P}$} & \multicolumn{2}{|c|}{$\mathrm{T}$} \\
\hline & & $\begin{array}{l}\text { Lat } \\
\left({ }^{\circ} \mathrm{N}\right)\end{array}$ & $\begin{array}{l}\text { Long } \\
\left({ }^{\circ} \mathrm{W}\right)\end{array}$ & $\begin{array}{l}\text { Prof. } \\
(\mathrm{km})\end{array}$ & & $\varphi$ & $\delta$ & $\lambda$ & $(\Phi)$ & $(\Theta)$ & $(\Phi)$ & $(\Theta)$ \\
\hline \multicolumn{13}{|c|}{ AMBIENTE I: ANTES DE LA FOSA } \\
\hline 4 & $13 / 12 / 1979$ & 2,88 & 79,49 & 15,0 & 6,3 & 207 & 72 & 84 & 302 & 27 & 108 & 63 \\
\hline 10 & $10 / 06 / 1985$ & 3,24 & 78,99 & 26,0 & 5,5 & 176 & 74 & 79 & 275 & 29 & 70 & 59 \\
\hline 11 & 25/01/1987 & 3,30 & 79,31 & 15,4 & 5,7 & 221 & 60 & -79 & 159 & 73 & 303 & 14 \\
\hline 19 & 03/06/1994 & 3,52 & 78,78 & 19,0 & 5,8 & 10 & 42 & -123 & 196 & 67 & 303 & 7 \\
\hline 21 & $26 / 11 / 1994$ & 2,87 & 79,43 & 15,9 & 5,3 & 206 & 57 & -73 & 159 & 72 & 284 & 10 \\
\hline 25 & $13 / 11 / 1995$ & 2,83 & 79,44 & 15,0 & 5,3 & 28 & 45 & -90 & 180 & 90 & 118 & 0 \\
\hline 35 & $21 / 12 / 2002$ & 3,76 & 78,90 & 15,0 & 5,2 & 36 & 40 & -70 & 50 & 76 & 292 & 7 \\
\hline 41 & 03/08/2005 & 2,62 & 79,40 & 28,6 & 4,9 & 34 & 35 & -64 & 54 & 70 & 285 & 13 \\
\hline 42 & $17 / 03 / 2007$ & 4,61 & 78,53 & 13,1 & 6,0 & 55 & 37 & -67 & 72 & 73 & 308 & 10 \\
\hline 43 & $18 / 03 / 2007$ & 4,69 & 78,53 & 12,0 & 6,2 & 38 & 36 & -69 & 64 & 74 & 294 & 10 \\
\hline 45 & $23 / 02 / 2008$ & 4,06 & 78,75 & 12,0 & 5,1 & 31 & 38 & -98 & 161 & 81 & 307 & 7 \\
\hline \multicolumn{13}{|c|}{ AMBIENTE II: ZONA DE FLEXIÓN E INTERACCIÓN INTERPLACAS } \\
\hline 1 & $16 / 02 / 1978$ & 4,00 & 78,00 & 14,0 & 5,6 & 53 & 55 & -100 & 289 & 78 & 150 & 9 \\
\hline 5 & 07/01/1980 & 2,94 & 78,77 & 30,8 & 5,3 & 185 & 77 & 84 & 280 & 31 & 87 & 58 \\
\hline 7 & 01/07/1980 & 2,93 & 78,75 & 19,0 & 5,3 & 185 & 77 & 84 & 280 & 31 & 87 & 58 \\
\hline 8 & 03/09/1980 & 3,24 & 78,19 & 42,1 & 6,1 & 186 & 70 & 92 & 275 & 25 & 99 & 65 \\
\hline 9 & 08/01/1982 & 2,57 & 78,87 & 25,1 & 5,3 & 171 & 76 & 73 & 247 & 29 & 59 & 56 \\
\hline 12 & 20/09/1988 & 4,99 & 77,68 & 24,1 & 5,8 & 179 & 73 & 85 & 272 & 27 & 82 & 62 \\
\hline 15 & 19/11/1991 & 4,80 & 77,18 & 14,9 & 7,2 & 188 & 77 & 89 & 279 & 32 & 97 & 58 \\
\hline 16 & 10/12/1991 & 4,74 & 77,48 & 38,4 & 5,2 & 186 & 72 & 76 & 287 & 26 & 76 & 60 \\
\hline 23 & 04/12/1995 & 2,72 & 78,32 & 49,4 & 5,1 & 65 & 53 & 127 & 129 & 2 & 36 & 61 \\
\hline 33 & $02 / 07 / 2002$ & 5,02 & 77,41 & 45,3 & 5,4 & 185 & 51 & 170 & 46 & 21 & 151 & 33 \\
\hline 34 & 08/08/2002 & 4,98 & 77,82 & 43,3 & 5,9 & 200 & 85 & 170 & 246 & 3 & 155 & 11 \\
\hline 36 & $22 / 08 / 2003$ & 3,07 & 77,73 & 31,4 & 5,0 & 50 & 47 & -106 & 246 & 78 & 152 & 1 \\
\hline 37 & $05 / 11 / 2003$ & 5,14 & 77,81 & 27,6 & 5,9 & 15 & 38 & -137 & 201 & 59 & 317 & 15 \\
\hline 38 & $15 / 11 / 2004$ & 4,70 & 77,51 & 15,0 & 7,2 & 177 & 79 & 85 & 271 & 34 & 81 & 55 \\
\hline 44 & $10 / 09 / 2007$ & 3,08 & 78,12 & 18,9 & 6,7 & 54 & 23 & -95 & 153 & 68 & 328 & 22 \\
\hline \multicolumn{13}{|c|}{ AMBIENTE III: CONTINENTAL SUPERFICIAL } \\
\hline 20 & 06/06/1994 & 2,93 & 75,94 & 32.6 & 6,8 & 206 & 76 & 170 & 72 & 3 & 163 & 17 \\
\hline 30 & 25/01/1999 & 4,58 & 75,75 & 27,7 & 6,1 & 8 & 65 & -21 & 329 & 32 & 236 & 4 \\
\hline 31 & 25/01/1999 & 4,31 & 75,74 & 24,3 & 5,5 & 17 & 67 & -23 & 337 & 32 & 246 & 2 \\
\hline
\end{tabular}


Cuadro 1 (continuación)

Parámetros de los mecanismos focales de los sismos localizados en los cuatro ambientes tectónicos estudiados, periodo 1976 - 2012.

\begin{tabular}{|c|c|c|c|c|c|c|c|c|c|c|c|c|}
\hline \multirow{3}{*}{ No. } & \multirow{3}{*}{$\begin{array}{c}\text { Fecha } \\
\text { dd:mm:aa }\end{array}$} & \multirow{2}{*}{\multicolumn{3}{|c|}{ Localización }} & \multirow{3}{*}{$\begin{array}{c}\text { Mag. } \\
\text { Mw }\end{array}$} & \multirow{2}{*}{\multicolumn{3}{|c|}{ Plano de falla }} & \multicolumn{4}{|c|}{ Ejes de deformación* } \\
\hline & & & & & & & & & \multicolumn{2}{|c|}{$\mathrm{P}$} & \multicolumn{2}{|c|}{$\mathrm{T}$} \\
\hline & & $\begin{array}{l}\text { Lat } \\
\left({ }^{\circ} \mathrm{N}\right)\end{array}$ & $\begin{array}{l}\text { Long } \\
\left({ }^{\circ} \mathrm{W}\right)\end{array}$ & $\begin{array}{l}\text { Prof. } \\
(\mathrm{km})\end{array}$ & & $\varphi$ & $\delta$ & $\lambda$ & $(\Phi)$ & $(\Theta)$ & $(\Phi)$ & $(\Theta)$ \\
\hline \multicolumn{13}{|c|}{ AMBIENTE IV: ZONA DE BENIOFF INTERMEDIA } \\
\hline 2 & $29 / 05 / 1979$ & 5,22 & 75,80 & 122,0 & 5,0 & 174 & 44 & -100 & 350 & 83 & 91 & 1 \\
\hline 3 & $23 / 11 / 1979$ & 4,81 & 76,22 & 105,4 & 7,2 & 137 & 41 & -163 & 342 & 42 & 95 & 24 \\
\hline 6 & $25 / 06 / 1980$ & 4,44 & 75,78 & 159,5 & 6,3 & 137 & 76 & 164 & 184 & 1 & 94 & 21 \\
\hline 13 & 29/11/1988 & 4,72 & 77,07 & 75,0 & 5,7 & 220 & 51 & 3 & 180 & 24 & 76 & 28 \\
\hline 14 & $23 / 11 / 1990$ & 4,73 & 75,60 & 138,0 & 6,1 & 53 & 75 & -72 & 129 & 28 & 346 & 56 \\
\hline 17 & $15 / 08 / 1992$ & 5,11 & 75,61 & 124,0 & 5,9 & 340 & 40 & -28 & 322 & 49 & 208 & 19 \\
\hline 18 & $12 / 10 / 1993$ & 4,15 & 76,98 & 102,9 & 5,2 & 234 & 44 & 77 & 154 & 2 & 53 & 81 \\
\hline 22 & 08/02/1995 & 4,10 & 76,62 & 77,1 & 6,4 & 30 & 69 & -90 & 300 & 66 & 120 & 24 \\
\hline 24 & $19 / 08 / 1995$ & 5,22 & 75,69 & 128,7 & 6,5 & 34 & 61 & -85 & 318 & 74 & 120 & 16 \\
\hline 26 & $11 / 09 / 1996$ & 4,59 & 76,90 & 118,2 & 5,3 & 61 & 63 & 45 & 181 & 7 & 280 & 50 \\
\hline 27 & $19 / 02 / 1997$ & 4,56 & 76,49 & 105,0 & 5,8 & 299 & 50 & 77 & 38 & 4 & 150 & 80 \\
\hline 28 & 02/09/1997 & 3,85 & 75,75 & 211,0 & 6,8 & 256 & 48 & 62 & 186 & 0 & 95 & 70 \\
\hline 29 & $11 / 12 / 1997$ & 3,93 & 75,79 & 183,0 & 6,3 & 140 & 66 & 139 & 197 & 8 & 99 & 46 \\
\hline 32 & $22 / 09 / 2001$ & 3,87 & 75,97 & 178,6 & 5,9 & 133 & 65 & 139 & 195 & 7 & 97 & 46 \\
\hline 39 & $08 / 03 / 2005$ & 4,50 & 75,92 & 64,5 & 5,0 & 171 & 79 & 159 & 219 & 6 & 127 & 22 \\
\hline 40 & $21 / 04 / 2005$ & 5,18 & 76,32 & 99,7 & 5,2 & 232 & 56 & 1 & 192 & 23 & 91 & 24 \\
\hline 46 & $13 / 09 / 2008$ & 4,79 & 75,52 & 132,9 & 5,7 & 72 & 79 & -85 & 349 & 56 & 158 & 33 \\
\hline 47 & 08/09/2009 & 4,77 & 76,71 & 82,7 & 4,8 & 60 & 49 & 66 & 166 & 2 & 261 & 72 \\
\hline 48 & $18 / 12 / 2009$ & 3,08 & 76,30 & 169,2 & 5,1 & 14 & 84 & -44 & 322 & 34 & 70 & 25 \\
\hline 49 & $29 / 01 / 2010$ & 4,83 & 76,07 & 133,4 & 5,0 & 352 & 51 & -105 & 206 & 77 & 93 & 5 \\
\hline
\end{tabular}

* La deformación en el medio se determina por el eje compresivo $P$ y el eje de tensión $T$, obtenidos del mecanismo focal. Las direcciones de estos ejes de deformación están definidos por los ángulos azimutales $\Phi_{P} \mathrm{y} \Phi_{T} \mathrm{y}$ los ángulos de buzamiento $\Theta_{P} \mathrm{y} \Theta_{T}$ Fuente de los datos: GCMT, ISC

$\theta$ es determinado por el ángulo entre la horizontal y la línea $U D . W_{x}$ y $W_{z}$ son las extensiones horizontal y vertical de la zona sismogénica (Conrad et al., 2004).

Considerando estos parámetros se determina el Ambiente tectónico II entre las coordenadas $2,08^{\circ}$ y $5,37^{\circ}$ de latitud Norte y $79,40^{\circ}$ y $77,68^{\circ}$ de longitud Oeste. Los datos de la solución del mecanismo de los terremotos se presentan en la Tabla 1 y su numeración se muestra en la Figura
5. Los sismos escogidos en este ambiente se ubican entre 15 y 50 kilómetros de profundidad. Este ambiente cubre un área de $39384 \mathrm{~km}^{2}$ y un volumen de $1378432 \mathrm{~km}^{3}$.

\section{Ambiente Tectónico III: Continental superficial}

Este ambiente se localiza entre las coordenadas $2,66^{\circ}$ y $5,59^{\circ}$ de latitud Norte y $77,44^{\circ}$ y 


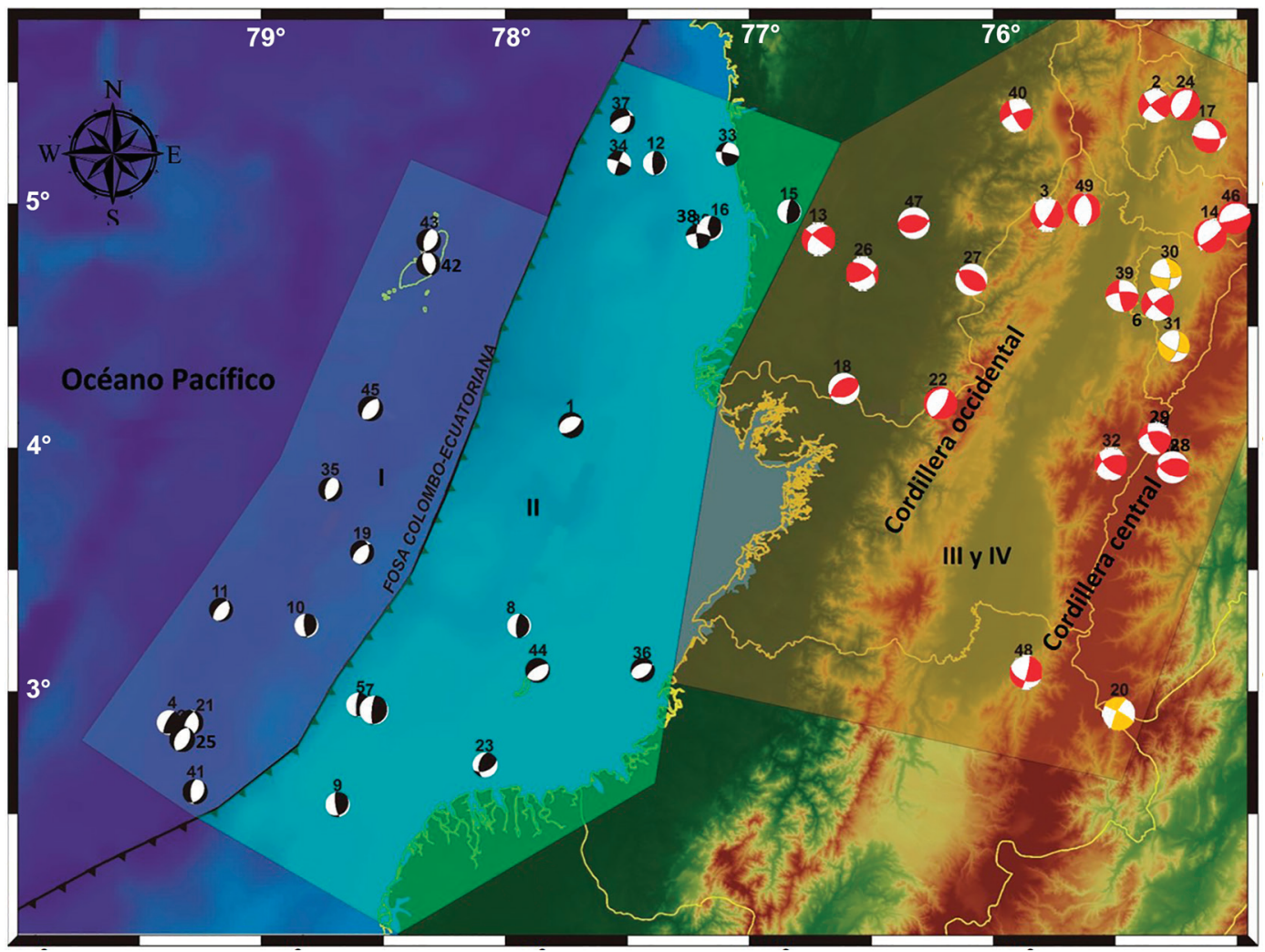

Fig. 5: Localización de los mecanismos focales de los terremotos utilizados en este estudio. Se muestran cuatro ambientes tectónicos determinados por los polígonos sombreados. Los mecanismos focales en naranja corresponden al ambiente III y en rojo al ambiente IV (modificado de Salcedo y Pérez, 2016).

$75,46^{\circ}$ de longitud Oeste, escogido en la parte de la corteza continental donde se presentan eventos superficiales. Los datos de la solución del mecanismo focal de los sismos se muestran en la Tabla 1, de igual manera, la numeración corresponde al número de cada uno de los eventos en la Figura 5. Los sismos utilizados se localizan hasta $32 \mathrm{~km}$ de profundidad y se distribuyen en un área de 56210 $\mathrm{km}^{2}$ con un volumen de $505892 \mathrm{~km}^{3}$.

\section{Ambiente Tectónico IV: Zona de Benioff intermedia}

Una vez que la litosfera se ha liberado de los esfuerzos generados en la zona de interacción, donde se nota un cambio drástico de sus parámetros cinemáticos y dinámicos, se produce un nuevo estado de esfuerzos que da origen a la ocurrencia de sismos de magnitud importante en el interior de la placa y a profundidad intermedia. La actividad sísmica en esta zona produce un nuevo régimen de deformación sismotectónica en la placa subducente.

Esta zona ha sido definida debajo del ambiente III, por lo que la profundidad de los sismos se ubica entre 64 y $211 \mathrm{~km}$. El área del ambiente IV es igual al del III pero el volumen equivale a $8206685 \mathrm{~km}^{3}$. Los datos de la solución del mecanismo de los sismos ocurridos en esta zona también se presentan en el cuadro 1. 


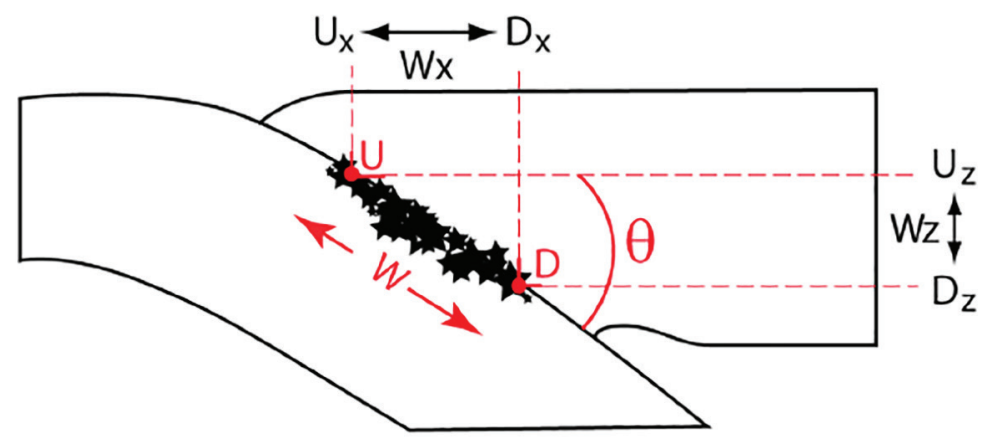

Fig. 6: Esquema de los parámetros que definen la geometría de la fuente sismogénica en la región de interfase de las placas en la zona de subducción. $U$ y $D$, definen los límites superior e inferior, respectivamente, de la inclinación de la región sismogénica; y sus coordenadas respectiva son $U_{x} ; U_{z}$ y $D_{x} ; D z$ medidas como las distancias máxima y mínima desde la trinchera o fosa y la profundidad. $W$ y $\theta$ corresponden a la extensión hacia debajo y ángulo de inclinación, respectivamente; mientras que $W_{x}$ y $W_{z}$, son las extensiones horizontal y vertical de la zona sismogénica (Modificado de Heuret et al., 2011).

\section{RESULTADOS Y DISCUSIÓN}

Considerando la división de la región de estudio en los cuatro ambientes tectónicos señalados anteriormente, se determinaron el tensor de velocidad de deformación y la velocidad media lineal de deformación sobre la base de las ecuaciones (1,2 y 3). Para cada ambiente tectónico, las componentes de la matriz de los vectores propios del tensor de momento sísmico son directamente proporcionales a los tres ejes principales de deformación $(T$, $N$ y $P$ ) (Jost y Herrmann, 1989; Stich et al., 2007; Guzmán-Speziale et al., 2005). Los resultados se muestran a continuación.

\section{Ambiente Tectónico I: Antes de la Fosa}

El tensor de momento sísmico es de la forma:

$$
M_{i j}=\left[\begin{array}{rrr}
-3.0260 & -0.7206 & -0.5687 \\
-0.7206 & 0.6718 & 1.2240 \\
-0.5687 & 1.2240 & 2.3570
\end{array}\right] * 10^{25} \text { dinas } \cdot \mathrm{cm}
$$

La matriz de vectores propios del ambiente I es:

$$
D=\left[\begin{array}{rrr}
-0.9844 & -0.1113 & -0.1364 \\
-0.1636 & 0.8644 & 0.4755 \\
-0.0650 & -0.4903 & 0.8691
\end{array}\right]
$$

El vector propio asociado a la extensión (eje $T$, primera columna) tiene un azimut de $9^{\circ}$ y una componente vertical (plunge) de $4^{\circ}$. El eje de compresión $P$ (tercera columna) es prácticamente vertical. En tanto el eje nulo o intermedio $N$ (segunda columna), es principalmente horizontal con orientación de $\mathrm{S} 83^{\circ} \mathrm{E}$. El vector propio asociado al valor propio más grande, es el eje $T$, lo que sugiere un ambiente dominante de extensión.

Puesto que este ambiente corresponde a la placa subducente, el coeficiente de viscosidad dinámico es igual a $\mu=6,5 \cdot 10^{23} \mathrm{~Pa} \cdot \mathrm{seg}$ (Dinther et al., 2010). El tensor de deformación es:

$$
\dot{\varepsilon}_{i j}=\left[\begin{array}{rrr}
-2.2090 & -0.5260 & -0.4150 \\
-0.5260 & 0.4900 & -0.8940 \\
-0.4150 & -0.8940 & 1.7210
\end{array}\right] * 10^{-13} a_{\tilde{n} o^{-1}}
$$

Los valores propios del tensor de deformación son:

$$
\dot{\varepsilon}_{i j}=\left[\begin{array}{r}
-2.3920 \\
-0.2000 \\
2.1940
\end{array}\right] * 10^{-13} a \tilde{n} o^{-1}
$$

Se tiene que la mayor tasa de deformación se presenta en dirección N-S con una magnitud de $2,39 \cdot 10^{-13}$ año ${ }^{-1}$ a una velocidad lineal de deformación de $8,6 \cdot 10^{-5} \mathrm{~mm} / \mathrm{año}$.

Usando la relación de magnitud $M w$ (4) y de deslizamiento promedio de ruptura del sismo 
(5), se obtiene que para este ambiente tectónico se esperaría un evento de magnitud de $6,6 \mathrm{Mw}$ y un deslizamiento promedio de ruptura de $\bar{D}=52 \mathrm{~cm}$.

\section{Ambiente Tectónico II: Zonas de Flexión y de interacción interplacas}

Los parámetros geométricos obtenidos para esta zona se muestran en el cuadro 2.

Puede observarse que el ángulo de inclinación $\beta=\theta=30^{\circ}$ es concordante con los valores reportados recientemente (Heuret et al., 2011; Martinod et al., 2010; Astiz et al., 1988; Arcila y Dimaté, 2005; Jarrard, 1986).

Considerando que la deformación en la zona responde a la de un ambiente de tipo visco-plástico, se calcula el tensor de deformación a partir de (2). El valor típico de $\mu$ en el límite de placas en la zona de subducción es $5,0 * 10^{11}$ dinas $\cdot \mathrm{cm}^{-2}$ (Kanamori y Astiz, 1985; Heuret et al., 2011).

El tensor momento sísmico es:

$$
M_{i j}=\left[\begin{array}{rrr}
0.4908 & 0.0642 & -1.3280 \\
0.0642 & 0.0931 & -0.0393 \\
-1.3280 & -0.0393 & -0.5831
\end{array}\right] * 10^{27} \text { dinas } \cdot \mathrm{cm}
$$

Los valores propios son:

$$
D=\left[\begin{array}{rrr}
0.9644 & -0.2630 & -0.0292 \\
0.2211 & 0.8614 & -0.4572 \\
-0.1454 & -0.4345 & 0.8889
\end{array}\right]
$$

El eje $T$ es principalmente horizontal con orientación de $\mathrm{N} 12^{\circ} \mathrm{E}$. El eje $P$ es prácticamente vertical. El eje intermedio $N$, es principalmente horizontal orientado $\mathrm{S} 73^{\circ} \mathrm{E}$. En este caso el vector propio asociando al valor propio más grande, es el eje $P$, lo que sugiere un ambiente dominantemente compresivo.

El tensor de la velocidad de deformación correspondiente es:

$$
\dot{\varepsilon}_{i j}=\left[\begin{array}{rrr}
4.9050 & -0.6410 & -13.2720 \\
-0.6410 & 0.9300 & -0.3930 \\
-13.2720 & -0.3930 & -5.8270
\end{array}\right] * 10^{-2} a \tilde{n}^{-1}
$$

Los valores propios del tensor de deformación son:

$$
\dot{\varepsilon}_{i j}=\left[\begin{array}{r}
-0.1481 \\
0.0095 \\
0.1386
\end{array}\right] * a \tilde{n} o^{-1}
$$

La mayor tasa de deformación se presenta en dirección N-S. La magnitud calculada para este ambiente es $M w=7,5$ y produciría un deslizamiento promedio de ruptura de $\bar{D}=150 \mathrm{~cm}$.

\section{Zona de flexión o punto de quiebre de la Placa subducente}

El esfuerzo tangencial deformante $\left(\tau_{s}\right)$ que actúa en la zona de flexión de la placa oceánica subducente se determina mediante la relación (11), donde $\varepsilon \tau=0,06 \cdot 10^{10}$ año ${ }^{-1}$. Por tanto, se tiene que:

$$
\tau_{s}=7,67 \cdot 10^{22} \mathrm{~Pa}
$$

Considerando el valor promedio de la litosfera como $H_{l}=30 \mathrm{~km}$, de acuerdo al espesor donde se presenta la actividad sísmica superficial, se obtiene que la fuerza tangencial $F_{s}(9)$, tiene un

\begin{tabular}{|c|c|c|c|c|c|c|c|c|}
\hline \multicolumn{3}{|c|}{ Coordenadas horizontal (km) } & \multicolumn{3}{|c|}{ Coordenadas vertical $(\mathrm{km})$} & \multirow{2}{*}{$\begin{array}{l}\text { Ext. } \\
\mathrm{W}\end{array}$} & \multirow{2}{*}{$\begin{array}{c}\text { Ángulo inclin. } \\
\theta=\beta\end{array}$} & \multirow{2}{*}{$\frac{\text { Long. fosa }}{(\mathrm{km})}$} \\
\hline Ux & Dx & Wx & $\mathrm{Uz}$ & $\mathrm{Dz}$ & Wz & & & \\
\hline 18,4 & 99 & 80,6 & 14 & 49,4 & 35,4 & 93,1 & $30^{\circ}$ & 369,41 \\
\hline
\end{tabular}
valor de:

Cuadro 2

Parámetros geométricos de la zona donde se presenta la interacción de la placa subducente y la placa continental en los límites del Valle del Cauca. Los símbolos se definen en la Figura 6. 


$$
F_{s}=1,06 \cdot 10^{27} \mathrm{~N} / \mathrm{m}
$$

Mientras que la fuerza normal Fn calculada a través de las ecuaciones (10) y (12), siendo

$$
\begin{gathered}
\sigma_{\mathrm{n}}=8,66 * 10^{24} \mathrm{~Pa}, \mathrm{es}: \\
F_{n}=1,47 \cdot 10^{28} \mathrm{~N} / \mathrm{m}
\end{gathered}
$$

La fuerza resultante (13), tiene un valor de:

$$
R=-R^{\prime}=1,57 \cdot 10^{28} \mathrm{~N} / \mathrm{m}
$$

Este valor corresponde a la fuerza de arrastre de las placas tectónicas en la zona de subducción, que como se mencionó arriba podría estar asociado a la posible ocurrencia de un sismo de magnitud $M w \geq 7$. Por razones naturales, comparativamente estos valores son mucho mayores que los obtenidos por Conrad et al. (2004) para la zona de subducción de América Central donde la fuerza de arrastre es de $1,77 \cdot 10^{16} \mathrm{~N} / \mathrm{m}$ con una fricción interplaca de $60 \%$.

\section{Velocidad de subducción de la placa}

Para el contexto geodinámico de la zona de subducción, es interesante determinar la velocidad de dislocación y la velocidad de hundimiento de la placa oceánica (ecuaciones 7 y 8). Considerando la velocidad de avance como $v_{l}=$ $5,8 \mathrm{~cm} /$ año (Comunicación personal Héctor Mora del Servicio Geológico de Colombia - Proyecto GEORED) y los valores de $\alpha$ y $\beta$ expresados arriba, se tiene que:

$$
\begin{aligned}
& v_{s}=3,68 \mathrm{~cm} / \text { año } \\
& v_{d}=2,67 \mathrm{~cm} / \text { año }
\end{aligned}
$$

Otro aspecto importante en el análisis sismotectónico es la estimación del acoplamiento interplaca, que es la capacidad entre dos placas para bloquear el rompimiento (resistencia o fricción), acumular esfuerzos y deformación. Para ello, se calculó el coeficiente de acoplamiento sísmico $\left(\chi_{\mathrm{s}}\right)$, el cual está definido como la relación entre la tasa de deslizamiento sísmico $\left(\mu_{s}\right)$ y la tasa de deslizamiento a largo plazo de la placa que subduce $\left(\mu_{m}\right)$ (Pacheco et al., 1993). La tasa de deslizamiento sísmico para un segmento de subducción rectangular determinada con $\mathrm{N}$ sismos más un período específico de tiempo (T) se puede expresar como:

$$
\mu_{s}=\sum_{n=1}^{N} \frac{M_{0}^{i}}{\mu L W T}
$$

Donde $\mu$ es la rigidez, L es la longitud del segmento, $\mathrm{W}$ es el ancho de la zona sismogénica, y $\mathrm{M}_{0}$ es el momento sísmico liberado individualmente por los terremotos (Brune, 1968; Pacheco et al., 1993), en este caso es el total del volumen de los ambientes tectónicos I y II, donde influye la zona de subducción a nivel superficial. Nosotros definimos la longitud de la fosa de la región de estudio en $369 \mathrm{~km}$ con un ancho de la zona sísmica inclinada de $93 \mathrm{~km}$ (ver Cuadro 2). El tiempo T, está definido para un periodo de 30 años que es lo que comprende la sismicidad en estos ambientes (1978-2008), en cuanto a $\mu_{m}$ lo asumimos igual al valor de $v_{d}$ que ya fue calculado. En este sentido, se tiene que el valor $\chi_{\mathrm{s}}$ es de 0,21 .

Para la mayoría de las zonas de subducción, el coeficiente de acoplamiento sísmico es menor que 1,0 (Pacheco et al., 1993). El valor de acoplamiento sísmico obtenido para nuestra región de estudio de 0,21 , que es relativamente bajo, y puede interpretarse como un débil acoplamiento asociado a una alta heterogeneidad y baja resistencia interplaca, lo que permite un mayor nivel de fracturamiento de la corteza generando mayor ocurrencia de sismos pequeños a moderados $(2 \leq M \leq 5)$, y con la posibilidad de que ocurran sismos de mayor magnitud (en promedio de magnitud 7,0 Mw, como se señaló anteriormente). El valor de acoplamiento sísmico de la zona de subducción del Valle del Cauca, puede ser comparado con el segmento del este de la región de Guerrero en México, cuyo valor es de 0,26 considerado como un valor de acoplamiento intermedio o de transición (difuso) entre las placas Rivera y Cocos. En éste segmento mexicano, los sismos grandes se encuentran entre 5,6 $M_{w}$ y 7,4 $M_{w}$ (Ávila-Barrientos et al., 2015), que son similares a los que ocurren en los ambientes I y II, que van desde 5,3 $M_{w}$ hasta 7,2 $M_{w}$. 


\section{Ambiente Tectónico III: Zona Continental superficial}

La deformación en esta zona corresponde a la de un ambiente cortical continental, por lo que el coeficiente de rigidez $\mu=3 \cdot 10^{11}$ dinas $\cdot \mathrm{cm}^{-2}$. Las componentes del tensor momento sísmico se expresan como:

$$
M_{i j}=\left[\begin{array}{rrr}
-0.098 & -0.722 & -0.153 \\
-0.722 & 1.400 & 0.833 \\
-0.153 & 0.833 & -1.303
\end{array}\right] * 10^{26} \text { dinas } \cdot \mathrm{cm}
$$

La matriz de vectores propios es:

$$
D=\left[\begin{array}{rrr}
0.5774 & -0.7009 & -0.4187 \\
0.8037 & 0.5782 & 0.1404 \\
0.1437 & -0.4176 & 0.8972
\end{array}\right]
$$

Se muestra que el vector propio asociado al eje de tensión $T$ es prácticamente horizontal y está orientado en la dirección $\mathrm{N} 54^{\circ} \mathrm{E}$. El eje de comprensión $P$ es básicamente vertical, mientras que el eje intermedio $N$ es principalmente horizontal con orientación de $\mathrm{S} 40^{\circ} \mathrm{E}$. El vector propio asociado al valor propio más grande, es el eje $P$, sin embargo, debido a la heterogeneidad de este ambiente que se refleja precisamente en su geomorfología (levantamiento de cordilleras) y rasgos estructurales (fallas en diferentes direcciones y formación de un graben), también se sugiere que hay interacción importante de procesos de extensión (deformación debido a $T$ ). Otra manera de comprender esto, es expresar los ejes de este ambiente en términos de su dimensión o magnitud, lo que constata la proporcionalidad de los mismos, corroborando lo sugerido, así, las componentes del tensor de velocidad de deformación son

$$
\dot{\varepsilon}_{i j}=\left[\begin{array}{rrr}
-0.643 & -4.760 & -1.007 \\
-4.760 & 9.226 & 5.488 \\
-1.007 & 5.488 & -8.583
\end{array}\right] * 10^{-13} \text { año }^{-1}
$$

Cuyos valores propios corresponden a:

$$
\dot{\varepsilon}_{i j}=\left[\begin{array}{r}
-2.7470 \\
-1.0500 \\
3.7970
\end{array}\right] * 10^{-13} a \tilde{n} o^{-1}
$$

La mayor tasa de deformación se presenta a nivel vertical lo que tiene sentido, pues es en este ambiente donde se dan los procesos de levantamiento de cordilleras y hundimiento tectónico. La velocidad lineal de deformación es de 3,8 $10^{-6} \mathrm{~mm} / \mathrm{año}$.

La magnitud que se podría esperar en este ambiente es de $\sim$, $0 \mathrm{Mw}$. La ruptura tendría un deslizamiento promedio de .

\section{Ambiente Tectónico IV: Zona de Benioff intermedia}

Como este ambiente corresponde a la zona intermedia de la placa subducida, el coeficiente de viscosidad dinámico es igual a $\mu=6,5 \cdot 10^{23}$ $\mathrm{Pa} \cdot$ seg. El tensor momento sísmico $\mathrm{M}_{\mathrm{ij}}$, se expresa como:

$$
M_{i j}=\left[\begin{array}{rrr}
-2.590 & -3.627 & -6.634 \\
-3.627 & -6.935 & 1.401 \\
-6.634 & 1.401 & 9.526
\end{array}\right] * 10^{26} \text { dinas } \cdot \mathrm{cm}
$$

Los vectores propios son:

$$
D=\left[\begin{array}{rrr}
-0.0390 & 0.9378 & 0.3449 \\
-0.2812 & 0.3209 & -0.9044 \\
0.9589 & 0.1323 & -0.2512
\end{array}\right]
$$

De tal manera que el eje $T$ tiene un azimut de $82^{\circ}$ y una componente vertical de $73^{\circ}$. El eje $P$ tiene una orientación de $\mathrm{S} 20^{\circ} \mathrm{E}$ y una componente vertical de $15^{\circ}$. El eje intermedio $N$, tiene una orientación de $\mathrm{N} 19^{\circ} \mathrm{E}$ con una componente vertical de $22^{\circ}$. Aquí, la dimensión o magnitud del eje $T$ es mayor, relacionando el ambiente con un medio extensivo. Esto es coherente con el hecho de que a profundidades entre 64 y $211 \mathrm{~km}$, comience un proceso de relajación de esfuerzos al interior de la placa que subduce.

Las componentes del tensor de velocidad de deformación son:

$$
\dot{\varepsilon}_{i j}=\left[\begin{array}{rrr}
-0.766 & -1.072 & -1.962 \\
-1.072 & -2.051 & 0.414 \\
-1.962 & 0.414 & 2.817
\end{array}\right] * 10^{-13} a \tilde{n} o^{-1}
$$


Y los valores propios son:

$$
\dot{\varepsilon}_{i j}=\left[\begin{array}{r}
-0.1015 \\
-0.0241 \\
0.1257
\end{array}\right] * 10^{-11} a_{\tilde{n} o^{-1}}
$$

La mayor tasa de deformación se presenta a nivel vertical, dado que en este ambiente se da el proceso de descenso de la placa lo que es consecuente con el resultado obtenido. La velocidad lineal de deformación es de $1,9 \cdot 10^{-4} \mathrm{~mm} / \mathrm{año}$.

Aquí la magnitud puede llegar a 7,4 Mw con un deslizamiento promedio de ruptura de $\bar{D}=1,3 \mathrm{~m}$.

De las mediciones de GPS realizadas entre 1996 y 2001, y ocupaciones posteriores realizadas en el 2003, se obtuvieron valores de tasa de deformación en el Valle del Cauca de 1,6 $10^{-}$ ${ }^{7}$ año $^{-1}$ a $2,1 \cdot 10^{-7}$ año $^{-1}$ (Trenkamp et al., 2004), cuya velocidad lineal de deformación es de $2,1 \cdot 10^{-6} \mathrm{~mm} /$ año. El valor estimado en este trabajo para esta zona (ambiente III) es de 3,8 $10^{-6}$ $\mathrm{mm} / \mathrm{año}$, de esta manera se puede deducir que los valores de deformación sismotectónica obtenidos a partir de datos de mecanismos focales guardan relación con los obtenidos con las mediciones GPS. Las diferencias que resultan entre estas estimaciones por ambos métodos, se deben a la diferencia entre los periodos de observación sísmica y los registrados por geodesia.

\section{CONCLUSIONES}

En concordancia con la distribución de los tipos de mecanismos focales de los terremotos fuertes que tienen lugar dentro de la región de estudio, se establece que el proceso de deformación de la zona suroeste de Colombia puede definirse en cuatro ambientes sismotectónicos: Zona lado marino de la fosa (Ambiente-I), Zona de flexión e interacción interplacas (AmbienteII), Zona continental superficial (Ambiente-III) y Zona de Benioff intermedia (Ambiente-IV). Estos ambientes se caracterizan por su propio régimen sísmico y estado de deformación sismotectónica.

El régimen de deformación del Ambiente-I es dominado por la extensión, donde el eje $T$ se orienta con un azimut de $9^{\circ} \mathrm{y}$ el eje de compresión es prácticamente vertical; mientras que eje intermedio $N$ principalmente horizontal con orientación de $\mathrm{S} 83^{\circ} \mathrm{E}$. Así, la mayor tasa de deformación se presenta en dirección N-S con una magnitud de $2,39 \cdot 10^{-13}$ año $^{-1}$ a una velocidad lineal de deformación de $8,6 \cdot 10^{-5} \mathrm{~mm} /$ año. Para este ambiente tectónico se esperaría un evento con magnitud de 6,6 $M w$ y un deslizamiento promedio de ruptura de .

El Ambiente-II se caracteriza por los procesos geodinámicos y la geometría que produce la interacción entre las dos placas (Nazca y Sudamérica) y el quiebre de la placa oceánica (Nazca), en este caso el régimen de deformación dominante es compresivo. El eje $T$ es principalmente horizontal con orientación de $\mathrm{N} 12^{\circ} \mathrm{E}$; el eje $P$ es prácticamente vertical y el eje intermedio $N$ es principalmente horizontal con orientación de $\mathrm{S} 73^{\circ} \mathrm{E}$. La mayor tasa de deformación se presenta en dirección N-S y se esperaría un evento con magnitud de 7,5 $\mathrm{Mw}$ y un deslizamiento promedio de ruptura de $\bar{D}=150 \mathrm{~cm}$.

Para la zona de flexión o punto de quiebre de la placa subducente, se observa que el ángulo $(\beta)$ con el cual la placa oceánica se inclina es de $30^{\circ}$. Por otro lado, el esfuerzo tangencial deformante $\left(\tau_{s}\right)$ es de $7,67 \cdot 10^{22} \mathrm{~Pa}$; la fuerza tangencial $\left(F_{s}\right)$ es de y la fuerza normal $\left(F_{n}\right)$ sería equivalente a, por lo que la fuerza de arrastre de la losa subducida es de $1,57 \cdot 10^{28} \mathrm{~N} / \mathrm{m}$. La velocidad lineal de deformación de la placa oceánica en esta zona es de $3,8 \cdot 10^{-6} \mathrm{~mm} /$ año y las velocidades de dislocación y hundimiento de la misma placa son de $v_{s}=3,68$ $\mathrm{cm} /$ año y $v_{d}=2,76 \mathrm{~cm} /$ año, respectivamente.

De acuerdo a los valores estimados de las fuerzas que actúan sobre la zona plástica y el coeficiente de acoplamiento sísmico $(0,21)$, la zona de subducción del suroeste de Colombia, se puede caracterizar como una zona de interacción intermedia. Esto explica la presencia de sismicidad de pequeña a moderada $(2 \leq M \leq 5)$ con mayor frecuencia, en comparación con la ocurrencia de eventos de mayor magnitud (en promedio de magnitud 7 $M w)$, que son menos frecuentes. Sin embargo, la poca presencia de sismos de mayor magnitud no significa un alivio, ya que este gap sísmico podría estar relacionado con una progresiva acumulación de esfuerzos en el tiempo, llevando al límite la deformación elástica interplaca, lo que posibilitaría 
desencadenar eventos de gran magnitud asociados con asperezas, como lo sugieren Condori-Quispe y Pérez (2016) para esta misma zona.

Para el ambiente III, el eje $T$ se orienta con un azimut de $54^{\circ}$ y una componente vertical de $10^{\circ}$; el eje $P$ tiene una orientación horizontal de $\mathrm{S} 71^{\circ} \mathrm{E}$ y una componente vertical de $81^{\circ}$; y el eje intermedio $N$ tiene una orientación de $\mathrm{S} 50^{\circ} \mathrm{E}$ con una componente vertical de $36^{\circ}$. En este caso el régimen de deformación mayor es de tipo compresivo, sin embargo, también el proceso de extensión o deformación debido a $T$ es importante, pues esto se refleja en los rasgos geomorfológicos y estructurales de la tectónica heterogénea de este ambiente. Por otro lado, la mayor tasa de deformación se presenta a nivel vertical lo cual se relaciona con los procesos de levantamiento de cordilleras y hundimiento tectónico. La magnitud de un terremoto que podría esperarse en este ambiente es de $6,8 \mathrm{Mw}$ con un deslizamiento promedio de ruptura de $\bar{D}=65 \mathrm{~cm}$.

En el ambiente IV los ejes de deformación están orientados así, el eje $T$ tiene un azimut de $82^{\circ}$ y una componente vertical de $73^{\circ}$; el eje $P$ tiene una orientación de $\mathrm{S} 20^{\circ} \mathrm{E}$ y una componente vertical de $15^{\circ}$; y el eje intermedio $N$, tiene una orientación de $\mathrm{N} 19^{\circ} \mathrm{E}$ con una componente vertical de $22^{\circ}$. En este caso, el régimen de deformación dominante es extensivo, lo cual es coherente con el hecho de que la sismicidad a profundidad intermedia de la placa que subduce, se asocia con el tipo de ruptura extensional que se genera por la descarga litostática debido a incidencia de la gravedad.

Dado que en este ambiente se da el proceso de descenso de la placa, la mayor tasa de deformación se presenta a nivel vertical con un valor de $0,12 \cdot 10^{-11}$ año-1 y una velocidad lineal de deformación de $1,9 \cdot 10^{-4} \mathrm{~mm} /$ año. La magnitud de un terremoto que podría esperarse en este ambiente es de 7,4 Mw con un deslizamiento promedio de ruptura de $\bar{D}=130 \mathrm{~cm}$.

\section{AGRADECIMIENTOS}

Los autores agradecen al doctor Walter Montero Pohly, por los comentarios y sugerencias que contribuyen a mejorar sustancialmente el contenido, la presentación y detalles técnicos de nuestro trabajo. Esta investigación fue realizada en el marco del proyecto "Caracterización del régimen sísmico y procesos de deformación cortical actual en el Valle del Cauca y zonas aledañas" financiado y registrado por la Vicerrectoría de Investigaciones de la Universidad del Valle con el código CI 297. Agradecemos al Observatorio Sismológico y Geofísico del Suroccidente Colombiano de la Universidad del Valle por apoyar este trabajo.

\section{REFERENCIAS BIBLIOGRÁFICAS}

Ávila-Barrientos, L., Zúñiga, F. R., RodríguezPérez, Q. y Guzmán-Speziale, M. (2015). Variation of $b$ and $p$ values from aftershocks sequences along the Mexican subduction zone and their relation to plate characteristics. Journal of South American Earth Sciences, 63, 162-171.

Aki, K. y Richards, P. (1980). Quantitative Seismology. Theory and Methods. San Francisco: Freeman.

Arcila, M. y Dimaté, C. (2005). Caracterización de fuentes sísmicas. En A. I. Alvarado y R. Daza (eds), Estudio de microzonificación sísmica de Santiago de Cali. Informe No. 1-6. Bogotá: INGEOMINAS-DAGMA.

Aspden, J. A. (1984). The geology of the Western Cordillera, Department of Valle, Colombia (Sheets 261, 278, 279, 280 y 299). Cali: INGEOMINAS - Misión Británica (British Geological Survey). Reporte No. 4 inédito.

Astiz, L., Thorne, L. y Kanamori, H. (1988). Large intermediate-depth earthquakes and the subduction process. Physics of the Earth and Planetary Interiors, 53(1-2), 80-166.

Brune, J. N. (1968). Seismic moment, seismicity and rate of slip along major fault zones. Journal of Geophysical Research, 73, 777-784. 
Capitanio, F. A., Goes, S., Morra, G. y Giardini, D. (2007). Signatures of downgoing platebuoyancy driven subduction in motions and seismic coupling at major subduction zones. Earth and Planetary Science Letters, 262, 298-306.

Collot, J. Y., Marcaillou, B., Sage, F., Michaud, F., Agudelo, W., Charvis, P. ... Spence, G. (2004). Are rupture zone limits of great subduction earthquakes controlled by upper plate structures? Evidence from multichannel seismic reflection data acquired across the northern Ecuadorsouthwest Colombia margin. Journal of Geophysical Research, 109(B11). doi:10.1029/2004JB003060

Conrad, C. P., Bilek, S. y Lithgow-Bertelloni, C. (2004). Great earthquakes and slab pull: interaction between seismic coupling and plate slab coupling. Earth and Planetary Science Letters, 218(1-2), 109-122.

Condori-Quispe, C. y Pérez, J. L. (2016). Análisis de la variación espacio-temporal del valor de "b" en el Valle del Cauca, Suroccidente de Colombia. Revista de la Unión Geofísica Mexicana. GEOS, 35(2), 1-13.

Corredor, F. (2003). Seismic strain rates and distributed continental deformation in the northern Andes and three-dimensional seismotectonics of northwestern South America. Tectonophysics, 372(3-4), 147166.

Dinther, Y. V., Morra, G., Funiciello, F. y Faccenna, C. (2010). Role of the overriding plate in the subduction process: Insights from numerical models. Tectonophysics, 484(1-4), 74-86.

Dziewonski, A. M. (1982). Harvard Centroid Moment Tensor Project. Recuperado de http://www.globalcmt.org.
Dziewonski, A. M. y Woodhouse, J. H. (1983). An experiment in systematic study of global seismicity: Centroid-moment tensor solutions for 201 moderate and large earthquakes of 1981. Journal of Geophysical Research, 88(B4), 3247-3271.

Dziewonski, A. M., Chou, T. A. y Woodhouse, J. H. (1981). Determination of earthquake source parameters from wave-form data for studies of global and regional seismicity. Journal of Geophysical Research, 86(B4), 2825-2852.

Dziewonski, A. M., Friedma, A., Giardini, D. yWoodhouse, J. H. (1983). Global seismicity of 1982: centroid-moment tensor solutions for 308 earthquakes. Physics of the Earth and Planetary Interiors, 33(2), 76-90.

Freymuller, J. T., Kellogg, J. N. y Vega V. (1993). Plate motions in the North Andean region. Journal of Geophysical Research, 98(B12), 21853-21863.

Guzmán-Speziale, M. (2001). Active seismic deformation in the grabens of northern Central America and its relationship to the relative motion of the North AmericaCaribbean plate boundary. Tectonophysics, 337(1-2), 39-51.

Guzmán-Speziale, M., Valdés-González, C., Molina, E. y Gómez, J. M. (2005). Seismic activity along the Central America volcanic arc: Is it related to subduction of the Cocos plate?. Tectonophysics, 400(1-4), 241-254.

Global CMT Catalog. (s.f.). Global CMT Catalog. Recuperado de http://www.globalcmt.org/

Gutsher, M.A., Malavieille, J., Lallemand, S. y Collot, J. Y. (1999). Tectonic segmentation of the North Andean margin: impact of the Carnegie Ridge collision. Earth and Planetary Science Letters, 168(3-4), 255-270. 
Heuret, A. y Lallemand, S. (2005). Plate motions, slab dynamics and back-arc deformation. Physics of the Earth and Planetary Interiors, 149(1-2), 31-51.

Heuret,A., Lallemand, S., Funiciello, F., Piromallo, C. y Faccenna, C. (2011). Physical characteristics of subduction interface type seismogenic zones revisited. Geochemestry, Geophysics, Geosystems, 12(1), Q01004. doi:10.1029/2010GC003230

International Seismological Centre (ISC). (s.f.). Recuperado de http://www.isc.ac.uk.

Jarrard, R. D. (1986). Relations among subduction parameters. Reviews of Geophysics, 24(2), 217-284

Jost, M. L. y Herrmann, R. B. (1989). A Student's Guide to and Review of Moment Tensors. Seismological Research Letters, 60(2), 37-57.

Kanamori, H. (1977). The energy release in great earthquakes. Journal of Geophysical Research, 82, 2981-2876.

Kanamori, H. (1986). Subduction-zone Earthquakes. Annual Review of Earth and Planetary Sciences, 14, 293-322.

Kanamori, H. y Astiz, L. (1985). The Akita-Oki Earthquake $(\mathrm{Mw}=7.8)$ and its implications for systematic of subduction earthquakes. Earthquake Prediction Research, 3, 305-317.

Kawasaki, I., Asai, Y., y Tamura, Y. (2001). Space-time distribution of interplate moment release including slow earthquakes and the seismo-geodetic coupling in the Sanriku-oki region along the Japan Trench. Tectonophysics, 330(3-4), 267-283.

Kellogg, J. N., Godley, V. M., Ropaín, C. A. y Bermudez, A. (1983). Gravity anomalies and tectonic evolution of northwestern
South America. Trabajo presentado en la 10a Conferencia Geológica del Caribe (pp. 18-31), Cartagena, Colombia.

Kostrov, B. (1974). Mecánica del foco de terremotos tectónicos. Moscú: Edit. Nauka (en ruso).

Kuchay, O. A. (1990). Deformaciones y desplazamientos de bloques de la corteza terrestres en zonas orogénicas intracontinentales (Mediante datos sobre mecanismos focales de terremotos). En Geodinámica de regiones montañosas intracontinentales (pp. 242-246). Nauka: Novosibirsk (en ruso).

Lobkovsky, L. I. (1988). Geodinámica de las zonas de expansión y subducción y de placas tectónicas superpuestas. Moscú: Nauka (En ruso).

López, M. C. (2006). Análisis de deformación tectónica en los pie de montes de las cordilleras Central y Occidental, Valle del Cauca, Colombia - Contribuciones Paleosísmicas (Tesis de maestría inédita). Universidad Eafit, Medellín, Colombia.

Martínez, M. (2015). Sismicidad histórica de la región del Valle del Cauca y zonas aledañas (Trabajo de grado inédito). Universidad del Valle, Cali, Colombia.

Martinod, J., Husson, L., Roperch, P., Guillaume, B. y Espurt, N. (2010). Horizontal subduction zones, convergence velocity and the building of the Andes. Earth and Planetary Science Letters, 299, 299-309.

Monsalve, H., 1998: Geometría de la subducción de la Placa Nazca en el noroeste de Colombia (Tesis de maestría inédita). Universidad Nacional Autónoma de México, México D.F., México. 
Nivia, A. (2001). Mapa Geológico del Departamento del Valle del Cauca (escala 1:250 000). Bogotá: INGEOMINAS. Memoria explicativa.

Nivia, A., Galvis, N. y Maya, M. (1997). Geología de la Plancha 242 - Zarzal. Bogotá: INGEOMINAS.

Pacheco, J. F., Sykes, L. R. y Sholz, C. H. (1993). Nature of seismic coupling along simple plate boundaries of subduction type. Journal of Geophysical Research, 98(B8), 14133-14159.

París, G., Marín, W., Sauret, B., Vergara, H. y Bles, J. L. (1992). Neotectónica. En CEEINGEOMINAS, Microzonificación sismogeotécnica de Popayán (pp. 28-49). CEEINGEOMINAS, Publicación Especial del INGEOMINAS N. 2.

Pedraza, P. (2006). Geometría de la subducción de la Placa Nazca en el suroeste de Colombia. Implicaciones tectónicas y sísmicas (Tesis de maestría inédita). Universidad Nacional de Colombia, Bogotá, Colombia.

Pennington, W. D. (1981). Subduction of the eastern Panama Basin and seismotectonics of northwestern South America. Jour. of Geophysical Research, 86(B11), 10753-10770.
Risnichenko, Yu. V. (1965a). Dinámica de la corteza terrestre. Moscú: Nauka (en ruso).

Riznichenko, Yu. V. (1965b). Relación entre el flujo sísmico de la masa rocosa y la sismicidad. Informes de la Académica de Ciencias de la URSS, 161(1), 97-99 (en ruso).

Risnichenko, Yu. V. (1976). Investigaciones sobre la física de los terremotos. Moscú: Nauka (En ruso).

Risnichenko, Yu. V. (1985). Problemas de la Sismología. Obras Escogidas. Moscú: Nauka (en ruso).

Risnichenko, Yu. V. y Jibladze, E. A. (1976). Velocidad de los movimientos verticales en el flujo sísmico de masas rocosas. Física de la Tierra, 1, 23-31 (en ruso).

Salcedo, E. De J. y Pérez, J. L. (2016). Caracterización sismotectónica de la región del Valle del Cauca y zonas aledañas a partir de mecanismos focales de terremotos. Boletín de Geología, 38(3), 89-107. doi: 10.18273/revbol.v38n32016006

Scholz, C. H. (1968). The frequency-magnitude relation of microfracturing in rock and 
its relation to earthquakes. Bulletin of the Seismological Society of America, 58(1), 399-415.

Stich, D., Martín, J. B. y Morales, J., 2007: Deformación sísmica y asísmica en la zona Béticas-Rif-Alborán. Revista de la Sociedad Geológica de España, 20(3-4), 311-319.

Trenkamp, R., Kellogg, J. N., Freymueller, J. T. y Mora-Páez, H. (2002). Wide plate margin deformation, southern Central America and northwestern South America, CASA GPS observations. Journal of South America Earth Science, 15, 157-171.
Trenkamp, R., Mora-Páez, H., Salcedo, E. y Kellogg, J. N. (2004). Possible rapid strain accumulation rates near Cali, Colombia, determined from GPS measurements (1996-2003). Earth Sciences Research Journal, 8(1), 25-33.

Turcotte, D. L. y Schubert, G. (1982). Geodynamics. Applications of continuum physics to geological problems. Nueva York: John Wiley and Sons.

USGS. (2000). Map of Quaternary Faults and Folds of Colombia and Its Offshore Regions (escala 1: 2500 000). Recuperado de https://pubs.usgs.gov/of/2000/ofr-000284/ofr-00-0284.plate.pdf 
